Document downloaded from:

http://hdl.handle.net/10251/84133

This paper must be cited as:

Ferrando Rocher, M.; Herranz Herruzo, Jl.; Valero-Nogueira, A.; Rodrigo Peñarrocha, VM. (2016). Circularly polarized slotted waveguide array with improved axial ratio performance. IEEE Transactions on Antennas and Propagation. 64(9):4144-4148. doi:10.1109/TAP.2016.2586492.

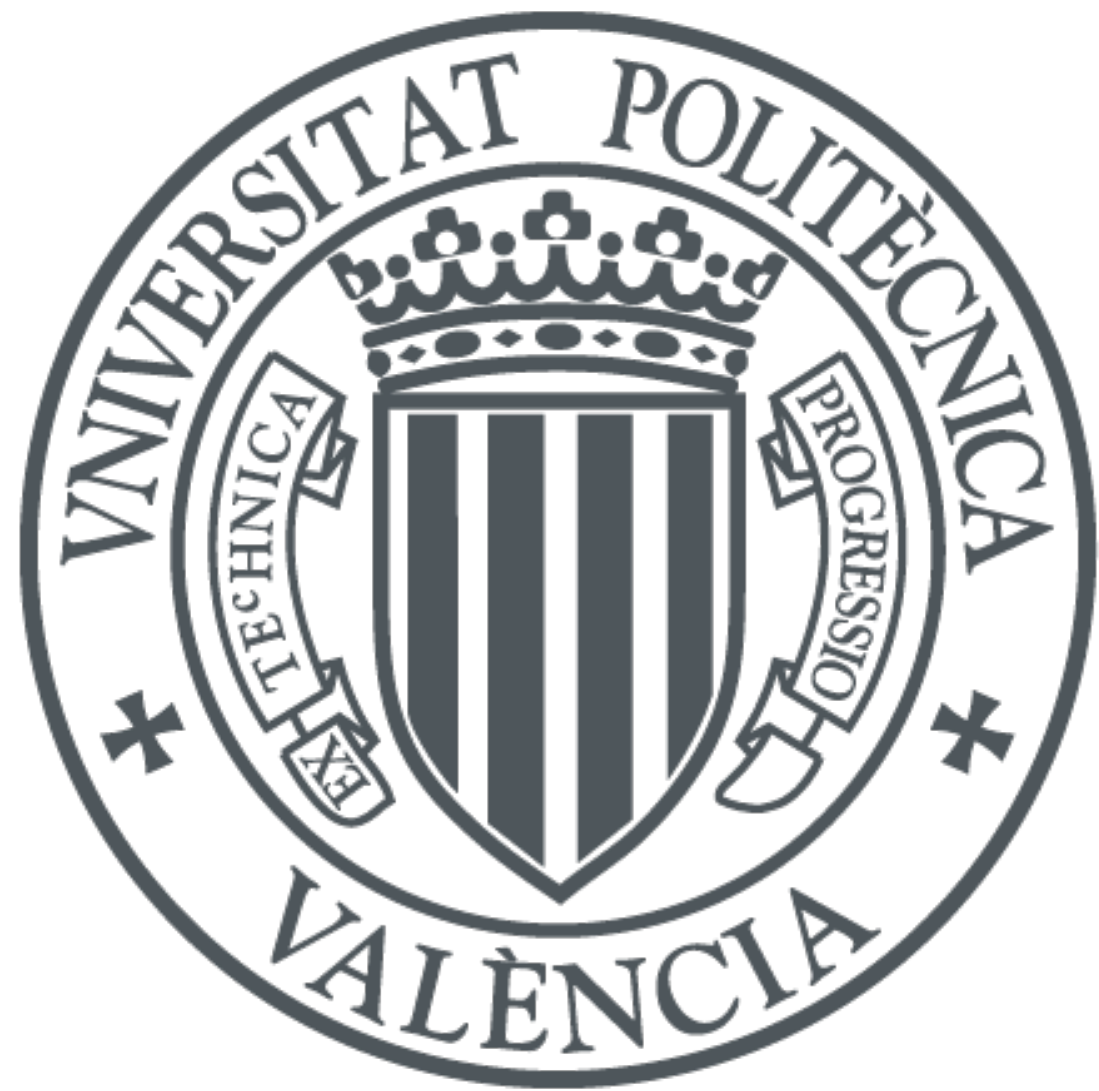

The final publication is available at

http://dx.doi.org/10.1109/TAP.2016.2586492

Copyright Institute of Electrical and Electronics Engineers (IEEE)

Additional Information 


\section{Circularly polarized slotted waveguide array with improved axial ratio performance}

\author{
Miguel Ferrando-Rocher, Student Member Jose \\ I. Herranz-Herruzo, Member Alejandro Valero-Nogueira, Senior \\ Member and Vicent Miquel Rodrigo
}

\begin{abstract}
This paper demonstrates experimentally the improvement in axial ratio performance when a slot is combined with a set of three parasitic dipoles instead of one. The analysis is performed in the context of slotted waveguide arrays and SATCOM applications in Ka band, where a bandwidth specification as wide as $7.5 \%$ can be demanded.
\end{abstract}

Keywords-Slotted-waveguide, Satellite communications, SOTM, Array, Mobile communications.

\section{INTRODUCTION}

Recently, a new generation of satellites offering multimedia services have been launched. These satellites operate in $\mathrm{Ka}$ band, offering a dramatic increase of the available capacity. However, requirements such as multi-spot coverage or circular polarization, as well as higher path loss pose important challenges with respect to existing platforms in $\mathrm{Ku}$ band. These antennas require a good polarization purity. This application calls for a wideband polarization performance, typically demanding an Axial Ratio (AR) below $1.5 \mathrm{~dB}$ within a $7.5 \%$ bandwidth.

Slotted-waveguide array antennas have been widely used in many radar and communications applications because of their geometric simplicity, efficiency, reliability and lightweight. Most slotted waveguide arrays are designed for linear polarization, although circular polarization can also be obtained by using crossed slots or combining radiation from tilted slots [1]. Nevertheless, linear-tocircular polarizers have been demonstrated in the literature, being meander-lines [2] the most popular ones. A single-layer dipole array has also been proposed as polarizer. In [3], it was theoretically shown that circular polarization could be attained by placing a tilted parasitic dipole at about $\lambda / 5$ above a slot. Two orthogonal field components are achieved by tilting the parasitic dipole a certain angle $\alpha$ with respect to the slot, while the phase quadrature is assured through the distance between slot and dipole. This technique has been successfully proved experimentally in a slotted waveguide array in [4]. This approach has advantages such as low profile, fabrication simplicity, good polarization purity and ease of design. The solution in [4], however, exhibited 3.6\% 3-dB-Axial-Ratio (AR) bandwidth, which is insufficient for the above-mentioned application. In [5] AR bandwidth was improved replacing the parasitic dipole by a parasitic patch. However cavities were needed to reduce mutual coupling and the overall design becomes much more complex. Interestingly, AR bandwidth can also be widened significantly through the addition of a second parasitic dipole, as explained in [3]. Indeed, the solution tried in this work uses three parasitic dipoles arranged as shown in Fig 1. This concept has already been proposed by these authors in [6]. In that paper preliminary results of a planar array combining slots and tilted dipoles were presented. In the present paper that early concept is investigated in detail and experimentally confirmed.

This work has been supported by the Spanish MINECO under project BES-2014-067917 and from the European Space Agency under contract 400010860913NLUS - project LOCOMO.

The authors are with the Instituto de Telecomunicaciones y Aplicaciones Multimedia (ITEAM) of the Universitat Politècnica de València (UPV), Cami de Vera s/n, 46022, Valencia, Spain

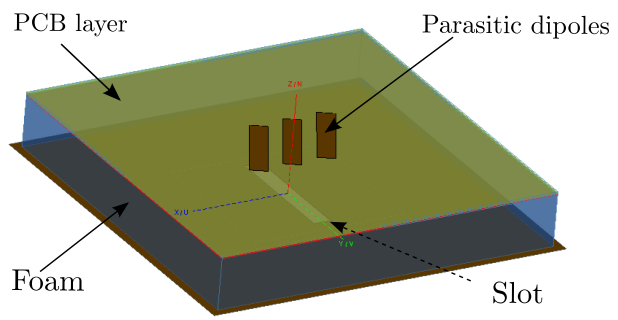

Fig. 1: Simplified model of the radiating element with three parasitic dipoles

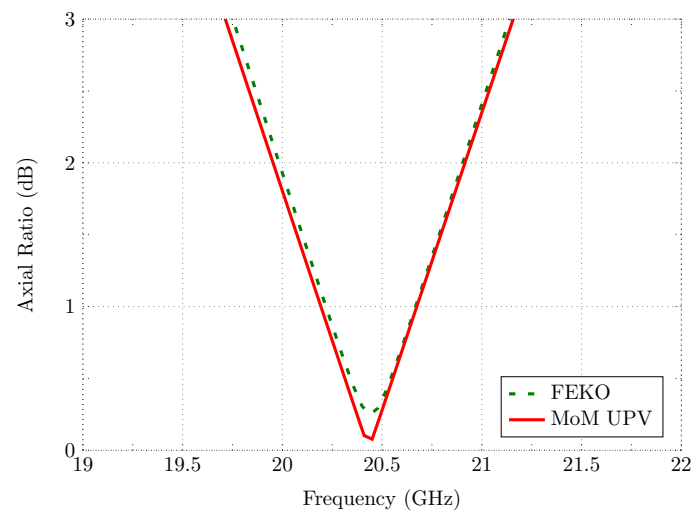

Fig. 2: AR performance of a slot-dipole pair.

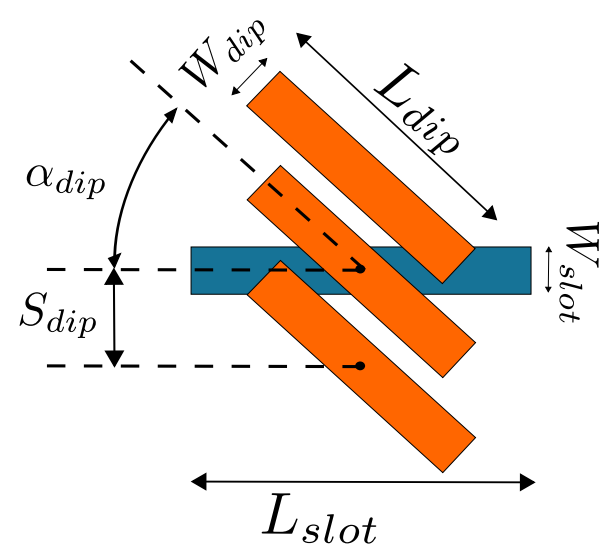

Fig. 3: Outline of the basic radiating element denoting all relevant dimensions.

\section{DESCRIPTION AND DESIGN OF THE BASIC RADIATING ELEMENT}

\section{A. Single parasitic dipole}

As mentioned before, one way to get circular polarization is by placing one or more dipoles above a slot and tilting them a certain angle. For the sake of reference, let us consider first the case of one parasitic dipole and set up the environment conditions which will be kept throughout the study. Center frequency is chosen to be $f_{0}=20.45 \mathrm{GHz}$. From a practical point of view, dipoles are usually etched in a thin substrate layer. In our case, substrate permittivity and thickness are $\epsilon_{r}=2.2$ and $t=127 \mu \mathrm{m}$, respectively. Such thin sheet rests on top of a spacer to provide the required height above the slot. Spacer permittivity should be as close to air as possible to 
TABLE I: Design parameters for slot and parasitic dipoles

\begin{tabular}{|c|c|c|c|}
\hline & 1 dipole & 2 dipoles & 3 dipoles \\
\hline$L_{\text {slot }}(\mathrm{mm})$ & \multicolumn{3}{|c|}{7.2} \\
\hline$W_{\text {slot }}(\mathrm{mm})$ & \multicolumn{3}{|c|}{500} \\
\hline$t_{\text {slot }}(\mu \mathrm{m})$ & \multicolumn{3}{|c|}{1} \\
\hline$L_{\text {dip }}(\mathrm{mm})$ & 5.427 & 5.1 & 4.909 \\
\hline$W_{\text {dip }}(\mathrm{mm})$ & \multicolumn{3}{|c|}{2.054} \\
\hline$t_{\text {dip }}(\mu \mathrm{m})$ & \multicolumn{3}{|c|}{36.58} \\
\hline$\alpha_{\text {dip }}$ & 33.71 & 35.52 & \\
\hline$S_{\text {dip }}(\mathrm{mm})$ & - & \multicolumn{3}{|c|}{} \\
\hline
\end{tabular}

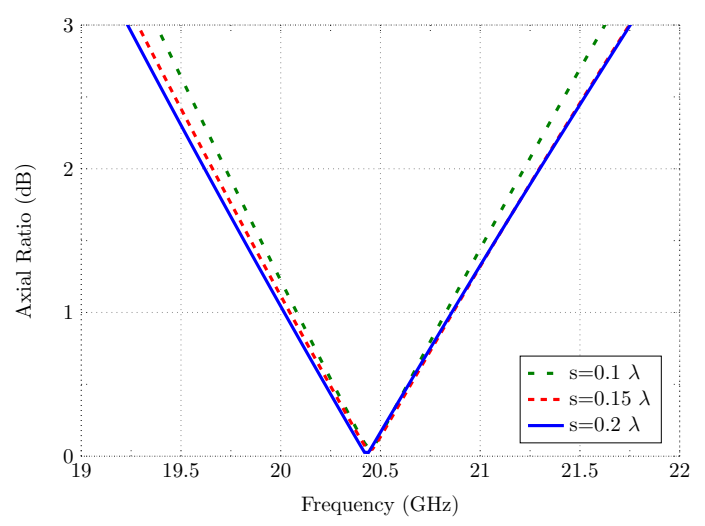

Fig. 4: Simulated Axial Ratio in the broadside direction vs frequency for several spacing between dipoles.

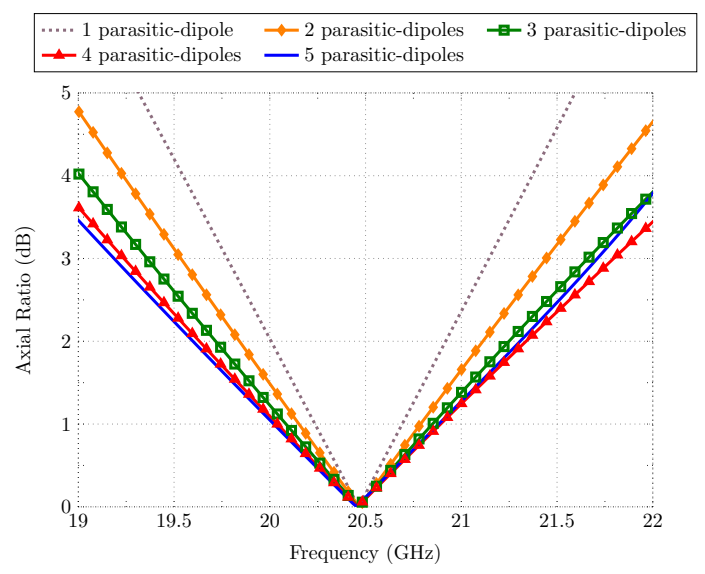

Fig. 5: Simulated Axial Ratio in the broadside direction vs frequency using $\mathrm{N}$ parasitic dipoles in the basic radiating element.

relax mutual coupling effects, which greatly compromise bandwidth. The chosen spacer is a 3 -mm thick Rohacell ${ }^{\circledR}$ foam with measured $\epsilon_{r}=1.08$. Once substrate and spacer are chosen, parasitic dipole length and tilt angle are optimized under such conditions to provide optimum circular polarization. Analysis and optimization have been performed using an inhouse Method of Moments code and optimizer [7]. Nevertheless FEKO [8] has been used as well for this first case as a benchmark. Achieved AR performance is shown in Fig. 2. It can be observed that $3-\mathrm{dB}$ bandwidth computed with our code practically matches that obtained with FEKO. This result consolidates the reliability of our MoM/Green's function code, which will be used in subsequent optimizations. The values of all relevant design parameters are detailed in Table I, whose meaning can be found in Fig. 3. Notice that $t_{\text {slot }}$ and $t_{d i p}$ represent slot and dipole thickness, respectively.

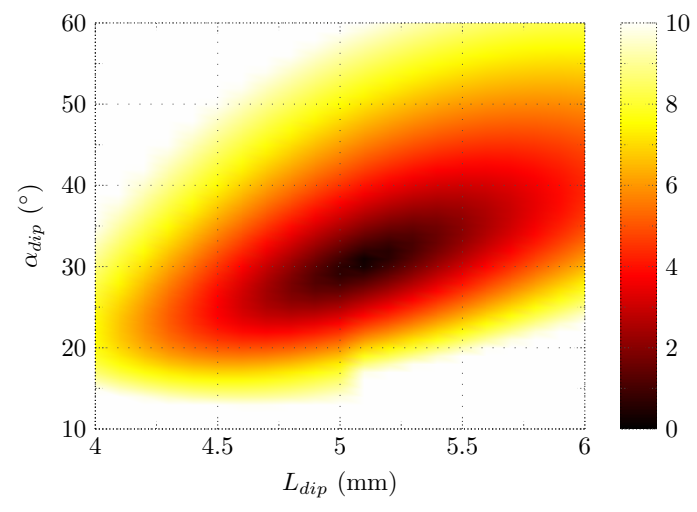

Fig. 6: Colormap showing Axial Ratio (dB) for a range of dipole lengths $\left(L_{d i p}\right)$ and tilting angles $\left(\alpha_{d i p}\right)$.

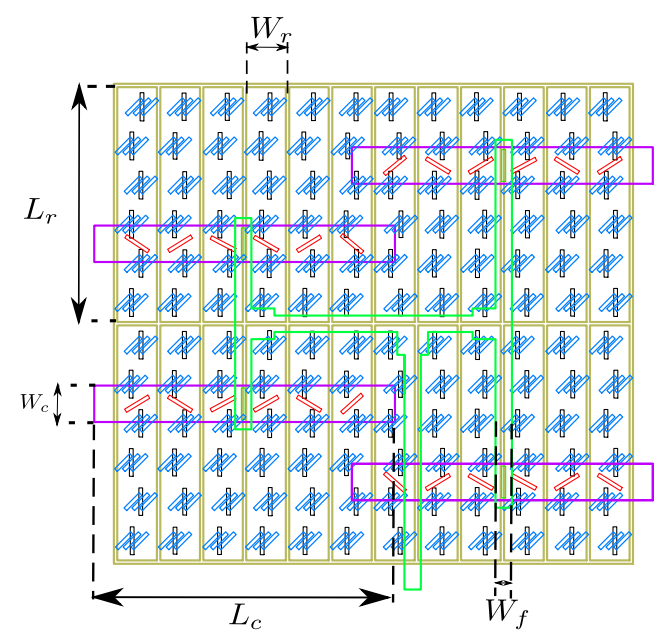

Fig. 7: Top view of the array antenna

\section{B. Multiple parasitic dipoles}

As anticipated in [3], the use of two parasitic dipoles per slot can widen the AR bandwidth with respect to one, though this fact has never been proved experimentally. Additionally, the work presented in [5] demonstrates that the adoption of wider parasitic patches improves the polarization bandwidth. In this case, parasitic dipoles are used instead, thus reducing transverse currents and facilitating the analysis. Let us first analyze in simulation the order of achievable AR improvement and whether more than two parasitic dipoles can provide even better performance. The configuration is shown in Fig. 1. As above, optimization is performed on the dipoles leaving slot dimensions fixed. Dipoles' length, width, tilting and spacing will be tuned up to an optimum AR in broadside direction for RightHand Circular Polarization (RHCP). All dipoles will be forced to share the same dimensions. In the case of two (or more dipoles) a new spacing variable, $S_{d i p}$, needs to be introduced. While parameter $S_{d i p}$ is not as decisive as dipole length or tilting, it may be adjusted roughly so as to achieve a larger polarization bandwidth. Even though Fig. 4 apparently reveals that the spacing between dipoles is not a particularly critical parameter, a compromise must be reached about its selection. On the one hand, dipoles must be spaced enough to assure a reliable fabrication without an eventual overlap. On the other hand, dipoles should not be too separated because it would increase mutual coupling with adjacent dipoles on nearby slots. Therefore, an intermediate distance of $s=0.14 \lambda_{0}$ has been chosen. 
TABLE II: Geometrical dimensions of the waveguides of each layer

\begin{tabular}{|l|c|c|c|c|c|c|c|}
\hline \multicolumn{7}{|c|}{ Feeding Layer } \\
\hline$H_{f}$ & $10.668 \mathrm{~mm}$ & $W_{f}$ & $4.318 \mathrm{~mm}$ & $L_{f}$ & - & $t_{f}$ & $0.5 \mathrm{~mm}$ \\
\hline \hline \multicolumn{7}{|c|}{ Coupling Layer } \\
\hline$H_{c}$ & $4.6 \mathrm{~mm}$ & $W_{c}$ & $9.61 \mathrm{~mm}$ & $L_{c}$ & $79.24 \mathrm{~mm}$ & $t_{c}$ & $0.5 \mathrm{~mm}$ \\
\hline \hline \multicolumn{7}{|c|}{ Radiating Layer } \\
\hline$H_{r}$ & $3.126 \mathrm{~mm}$ & $W_{r}$ & $10.41 \mathrm{~mm}$ & $L_{r}$ & $61.87 \mathrm{~mm}$ & $t_{r}$ & $0.5 \mathrm{~mm}$ \\
\hline
\end{tabular}

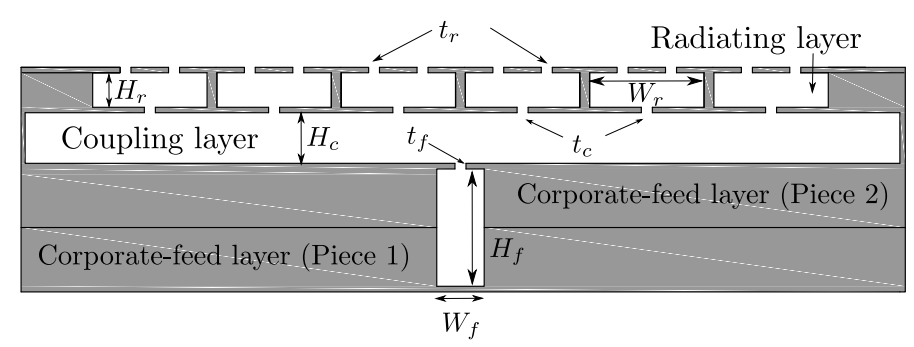

Fig. 8: Side view of the antenna excluding the polarizing layer which is explained in detail in Fig. 9.

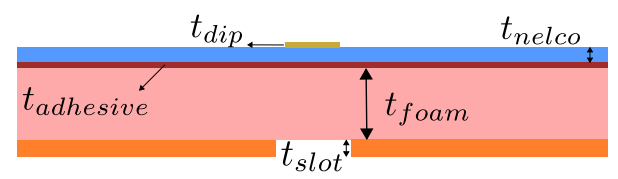

Fig. 9: Side view of the polarizing layer above the radiating slots.

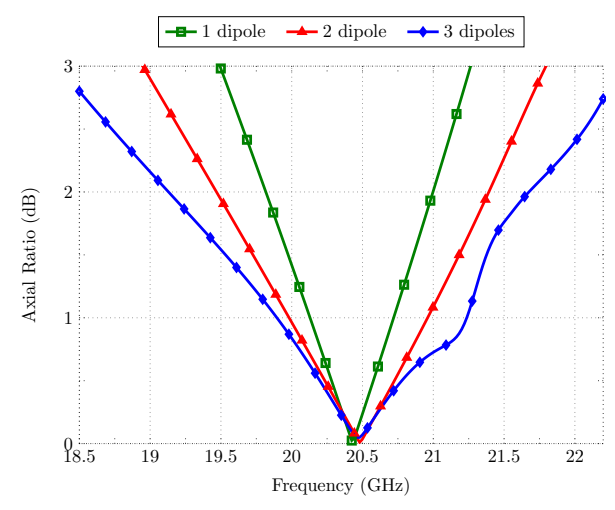

Fig. 10: Simulated AR bandwidth (MoM) for a varying number of parasitic dipoles in the array environment.

Regarding the number of parasitic elements, the optimized frequency performance of AR for one to five dipoles is shown in Fig. 5 for comparison. Two parasitic dipoles provide a remarkable improvement with respect to one. The 3-dB AR bandwidth is around $9.8 \%$ with a pair of dipoles instead of the $7 \%$ of a single dipole. In addition, the three-dipole case provides even further improvement, reaching a value of $12 \%$ in relative bandwidth. Cases with four and five dipoles do not offer a noteworthy improvement, since outermost dipoles are not efficiently illuminated by slot's near field. Lastly, colormap in Fig. 6 shows the AR for a set of dipole lengths, $L_{d i p}$, and tilting angles, $\alpha_{\text {dip }}$. It is observed that a good AR can be achieved for a wide range of lengths and tiltings. This behavior facilitates the design process and suggests that this solution will be robust against tolerance errors.
TABLE III: Simulated parameters of the prototype.

\begin{tabular}{|c|c|c|c|c|c|}
\hline Frequency $(\mathrm{GHz})$ & 19.7 & 20.075 & 20.45 & 20.8 & 21.2 \\
\hline \hline Axial Ratio $(\mathrm{dB})$ & 1.13 & 0.72 & 0.11 & 0.5 & 1.18 \\
\hline Directivity $(\mathrm{dBi})$ & 28.99 & 29.27 & 29.60 & 29.48 & 28.54 \\
\hline
\end{tabular}

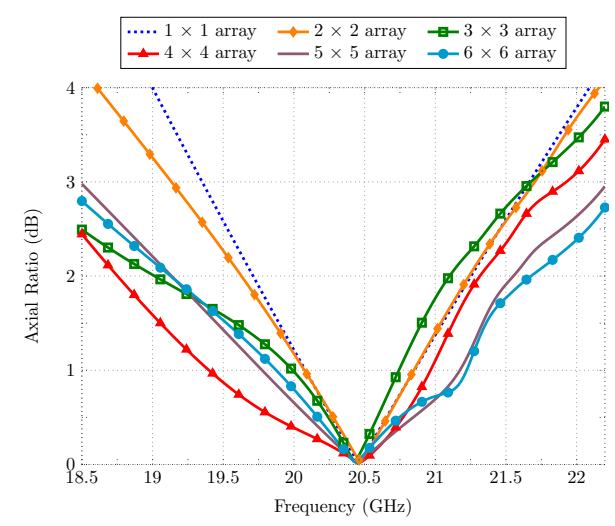

Fig. 11: Simulation of a slot planar array of NxN slots combined with 3-parasitic dipoles per slot.

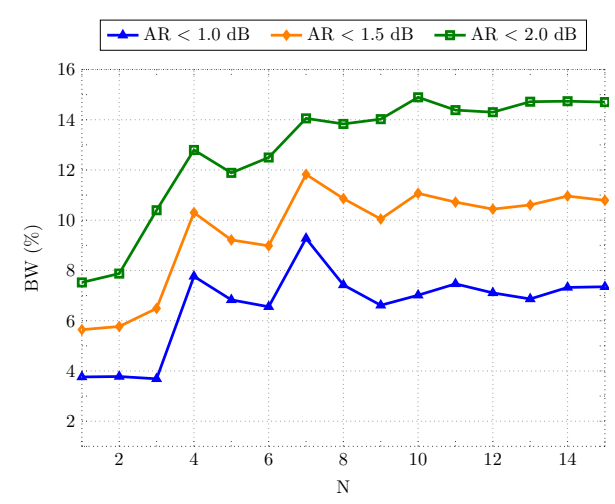

Fig. 12: Axial Ratio bandwidth in a $\mathrm{NxN}$ array.

\section{PERFORMANCE OF THREE PARASITIC DIPOLES PER SLOT IN A FULL ARRAY}

In this section, the study is extended to a full array formed by several slot-dipoles elements. The targeted antenna, sketched in Fig. 7 , comprises four subarrays, each one composed by $6 \times 6$ elements. The subarray arranges the slots in a conventional standing-wave configuration. Each shunt slot exhibits its own length and offset in order to meet the given matching and illumination requirements.

The optimization method compensates mutual coupling so that maximum AR bandwidth is achieved while assuring resonance for all 36 slots synthesizing a uniform distribution. The following dimensions has been obtained: $L_{d i p}=6.03 \mathrm{~mm}, W_{d i p}=1 \mathrm{~mm}, W_{\text {slot }}=1 \mathrm{~mm}$, $\alpha_{\text {dip }}=39^{\circ}$. Notice that dipole dimensions are kept uniform for all the array elements. Achieved AR performance can be observed in Fig. 10 , which also includes single and double-dipole case for comparison purposes. This graph reveals a $3-\mathrm{dB}$ AR bandwidth of $17 \%$ for the optimum 3-dipole case, which is twice as much as that achieved for a single parasitic dipole $(8.1 \%)$. To illustrate more clearly the link between AR bandwidth and element arraying, Figs. 11 and 12 plot $\mathrm{AR}$ vs. frequency and $\mathrm{AR}$ bandwidth vs. number of elements in an array, respectively. Both figures confirm the advantageous effect of mutual coupling for AR bandwidth. Furthermore, Fig. 12 shows that 
TABLE IV: Measured parameters of the prototype.

\begin{tabular}{|c|c|c|c|c|}
\hline Frequency (GHz) & 19.7 & 20.075 & 20.45 & 21.2 \\
\hline \hline Axial Ratio (dB) & 0.65 & 0.33 & 0.95 & 1.1 \\
\hline Directivity (dBi) & 28.89 & 29.29 & 29.31 & 28.09 \\
\hline Aperture efficiency (\%) & 86.06 & 85.59 & 88.58 & 56.76 \\
\hline Gain (dBi) & 27.62 & 28.01 & 27.91 & 26.48 \\
\hline Antenna efficiency (\%) & 74.65 & 74.31 & 72.32 & 68.94 \\
\hline
\end{tabular}

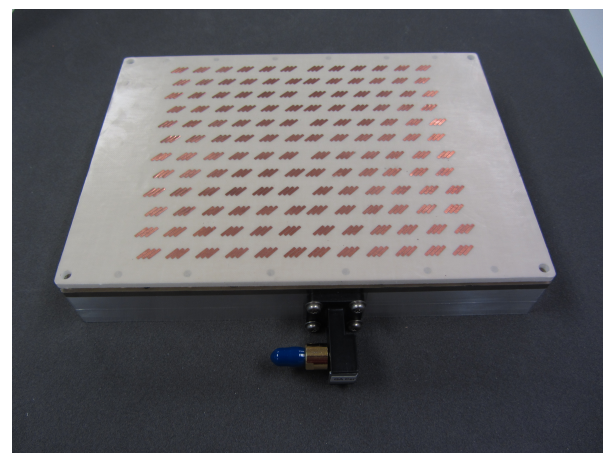

Fig. 13: Manufactured prototype

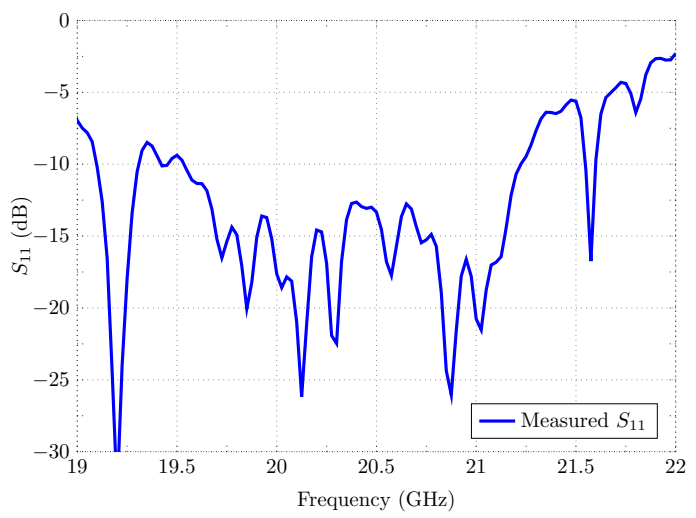

Fig. 14: Measured reflection coefficient at input port

frequency performance is nearly stabilized for arrays larger than $4 \times 4$ elements.

\section{EXPERIMENTAL RESULTS}

A slotted waveguide array including parasitic dipoles has been designed and fabricated to test the performance of the radiating element. A sketch of the array is shown in Fig. 7. It is composed of 4 subarrays like the one analyzed in previous section. The four subarrays are fed with a corporate feeding network providing uniform weighting. The antenna is aimed to cover the band from 19.7 to 21.2 GHz. The total number of radiating slots is 144 and the total size of the antenna is $13 \times 13 \mathrm{~cm}^{2}$. A complete sidecut of the power distribution network is presented in Fig. 8. Meaningful dimensions are gathered in Table II. An accurate modelling of the layered media supporting the parasitic dipoles is key to provide reliable results. Fig. 9 shows a side view of the layered media. As mentioned above, a Rohacell foam spacer is placed between radiating slots layer and the substrate with the etched dipoles. The thickness of the foam is $3 \mathrm{~mm}$ and its permittivity $\epsilon_{r 1}=1.08$. The bonding material was also taken into account in the simulations. This layer is $70-\mu \mathrm{m}$ thick with a permittivity of $\epsilon_{r 2}=2.28$. It is worth remarking that these two materials have been electrically characterized by an adhoc

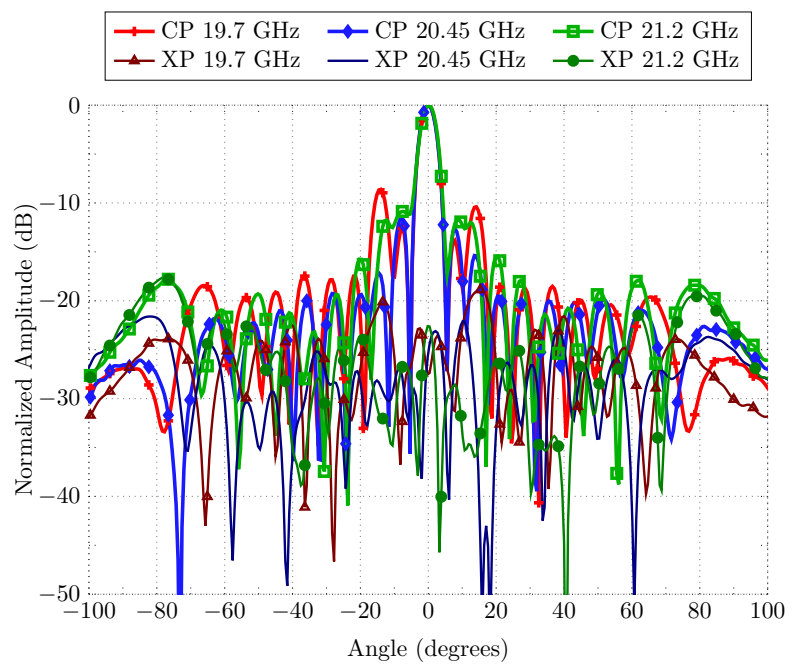

(a)

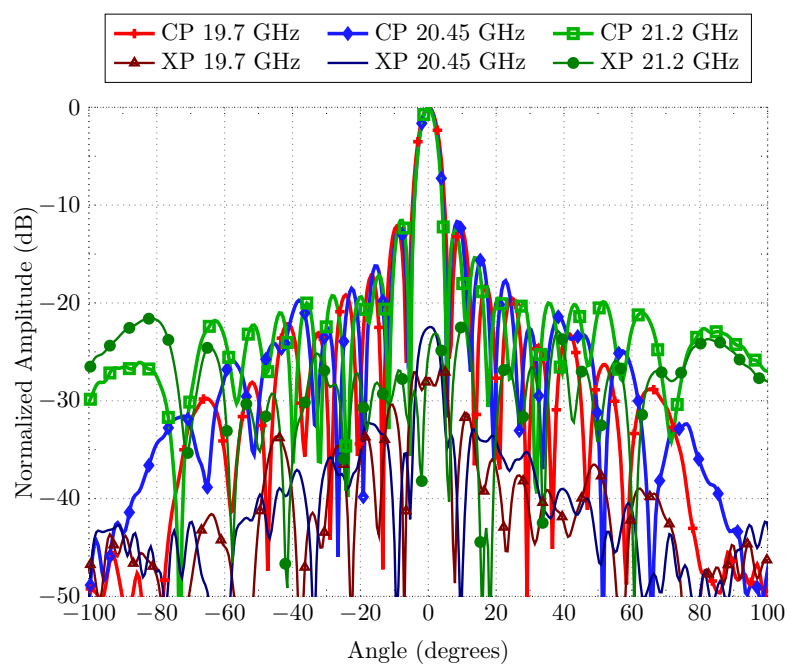

(b)

Fig. 15: (a) Measured copolar and cross-polar radiation pattern in the elevation plane. (b) Measured copolar and cross-polar radiation pattern in the azimuth plane.

measurement setup. Finally, the parasitic dipoles are etched on a substrate of thickness $127 \mu \mathrm{m}$ and $\epsilon_{r 3}=2.2$. The simulated parameters achieved after the optimization process can be seen in Table III. As mentioned this is a breadboard that is a quarter of the final antenna panel. The purpose of that antenna is to be used for satellite onthe-move applications. Details and dimensions of the full antenna may be found in [6]. Fig. 13 shows a picture of the fabricated prototype. The antenna was measured in an anechoic chamber. Fig 14 shows the $S_{11}$-parameter of the antenna. Figs. 15a and $15 \mathrm{~b}$ show the copolar and cross-polar components of the radiation pattern at several representative frequencies in the target band. Patterns in agreement with target uniform illumination can be seen for both main cuts. Cross-polar patterns, show levels below $-20 \mathrm{~dB}$ for both cuts in the whole band. Such a low cross-polar radiation guarantees a wideband polarization performance. Notice that the elevation cut, Fig. 15a, is the one perpendicular to the slots in our reference. Table IV displays measured AR, directivity and aperture efficiency values for several frequencies within the band of interest. Although it was not the main objective of the project, gain and antenna efficiency are also shown. 
Antenna efficiency values are not as good as expected due to the low conductivity of the nickel used for plating the two pieces forming the radiating layer (Fig. 8). These values could be improved using silver plating. Regarding AR, excellent performance is achieved, below 1.2 dB. Consequently, the proposed solution succeeds in broadening the typical bandwidth reached with this kind of polarizers. As a result, a practically flat frequency performance in AR has been reached along a considerable bandwidth, 1.1-dB AR for $7.5 \%$ bandwidth.

\section{CONClusions}

The improvement in AR bandwidth through the use of more than one parasitic dipole above a slot has been experimentally demonstrated. Successful measurement results have relied on a careful modelling of layered media, including bonding layer, and a precise knowledge of electric properties of all materials involved.

\section{ACKNOWLEDGEMENTS}

The authors would like to acknowledge the contribution of Professors J.A. Encinar and M. Barba in selecting the bonding material for the dielectric layers.

\section{REFERENCES}

[1] G. Montisci, M. Musa, and G. Mazzarella, "Waveguide slot antennas for circularly polarized radiated field," IEEE Transactions on Antennas and Propagation,, vol. 52, no. 2, pp. 619-623, Feb 2004.

[2] L. Young, L. Robinson, and C. Hacking, "Meander-line polarizer," IEEE Transactions on Antennas and Propagation, vol. 21, no. 3, pp. 376-378, May 1973.

[3] K. Itoh and T. Adachi, "Novel circularly polarized antennas combining a slot with parasitic dipoles," in Antennas and Propagation Society International Symposium, 1980, vol. 18, June 1980, pp. 52-55.

[4] K.-S. Min, J. Hirokawa, K. Sakurai, M. Ando, and N. Goto, "Singlelayer dipole array for linear-to-circular polarisation conversion of slotted waveguide array," Microwaves, Antennas and Propagation, IEEE Proceedings, vol. 143, no. 3, pp. 211-216, Jun 1996.

[5] Y. Li, Z. N. Chen, X. Qing, Z. Zhang, J. Xu, and Z. Feng, "Axial ratio bandwidth enhancement of 60 -ghz substrate integrated waveguide-fed circularly polarized ltcc antenna array," IEEE Transactions on Antennas and Propagation, vol. 60, no. 10, pp. 4619-4626, Oct 2012.

[6] J. Herranz-Herruzo, A. Valero-Nogueira, M. Ferrando-Rocher, B. Bernardo-Clemente, R. Lenormand, A. Hirsch, J.-L. Almeida, M. Arnaud, and L. Barthe, "Low cost switchable RHCP/LHCP Antenna for SOTM Applications in Ka-band," in 9th European Conference on Antennas and Propagation (EuCAP), 2015, April 2015.

[7] D. Gonzalez-Ovejero, J. Herranz-Herruzo, A. Valero-Nogueira, and J. Balbastre-Tejedor, "Design of radome-covered slot-array antennas loaded with parasitic dipoles for circular polarization at ka band," in The Second European Conference on Antennas and Propagation, 2007. EuCAP 2007., Nov 2007, pp. 1-6.

[8] Altair Development S.A. (Pty), FEKO Suite 7.0, http://www.altairhyperworks.com, Stellenbosch, South Africa. 